\title{
Mesoscopic light transport by very strong collective multiple scattering in nanowire mats.
}

\author{
Tom Strudley, ${ }^{1}$ Tilman Zehender, ${ }^{2}$ Claire Blejean, ${ }^{1}$ \\ Erik P. A. M. Bakkers, ${ }^{2,3}$ and Otto L. Muskens*1 \\ ${ }^{1}$ Faculty of Physical and Applied Sciences, \\ University of Southampton, Highfield, \\ Southampton SO17 1BJ, United Kingdom. \\ ${ }^{2}$ Department of Applied Physics, Eindhoven University of Technology, \\ P.O. Box 513, 5600MB, Eindhoven, The Netherlands. \\ ${ }^{3}$ Kavli Institute of Nanoscience, Delft University \\ of Technology, 2600 GA Delft, The Netherlands.
}

(Dated: July 14, 2013)

\begin{abstract}
Under the extreme condition of the scattering length being much shorter than the wavelength, light transport in random media is strongly modified by mesoscopic interference, and can even be halted in an effect known as Anderson localization. Anderson localization in three dimensions has recently been realized for acoustic waves and for cold atoms. Mats of disordered, high-refractive index semiconductor nanowires are one of the strongest three-dimensional scattering materials for light, but localization has not been shown. Here, we use statistical methods originally developed for microwave waveguides to demonstrate that transport of light through nanowire mats is strongly correlated and governed by mesoscopic interference contributions. Our results confirm the contribution of only a few open modes to the transmission.
\end{abstract}


Mesoscopic physics deals with wave interference effects that occur on a scale between that of individual microscopic scatterers and the macroscopic average medium ${ }^{1,2}$. Every scattering medium has a finite number of ingoing and outgoing degrees of freedom, defined by the amount of diffraction limited spots at maximum numerical aperture per unit of area. The transmission of the medium is then described by a matrix, with elements $t_{a b}$ being the transmission from an ingoing channel $a$ to an outgoing channel $b$. A crucial result from random matrix theory is that the eigenmodes of the transmission matrix are not uniformly distributed. Instead, they consist of a large number of "closed" modes with negligible transmission and only a limited amount of "open" modes with a transmission close to unity. The average number of open modes is equivalent to the dimensionless conductance, $g=\sum_{a, b} t_{a b}$. Recently wavefront shaping techniques have enabled direct measurement of (parts of) the transmission matrix ${ }^{3}$ and control over individual transmission eigenmodes of complex photonic media ${ }^{4,5}$.

While recent experiments have been successful in demonstrating the existence of open transport channels, the regime of strong light scattering and, in particular, the transition from diffuse transport to strong interference and Anderson localization in three dimensional media is still an open problem ${ }^{6}$. Anderson localization in three dimensions has only recently been realized in acoustic wave experiments ${ }^{7}$ and cold atoms ${ }^{8}$, convincingly demonstrating that mesoscopic transport is a general wave phenomenon. Pioneering studies have reported indications of three-dimensional localization of light ${ }^{9,10}$. However, their conclusions were based on the properties of the average intensity, where it is difficult to separate localization effects from other contributions such as absorption or fluorescence ${ }^{11}$. Demonstrations of mesoscopic interference corrections in three-dimensional materials in the optical regime ${ }^{12,13}$ have been limited to values of the conductance $g$ in the range $10^{2}-10^{4}$.

Here we demonstrate very strong, collective multiple scattering and mesoscopic light transport in three dimensional nanowire mats. Understanding light transport in these nanowire mats is of considerable importance for applications in next-generation light harvesting and optoelectronics devices ${ }^{14-17}$. The nanowire mats studied here consist of a high density of GaP nanowires grown epitaxially on a GaP (100) substrate using a combination of vapor-liquid-solid growth and sidewall growth (see Method). Importantly, samples can be fabricated with high uniformity over areas of several $\mathrm{cm}^{2}$ and exhibit negligible absorption of wavelengths longer than the indirect electronic band gap at $548 \mathrm{~nm}$. Figure 1(a) shows a 
scanning electron microscopy (SEM) image of a cleaved section of the 6 - $\mu$ m thick nanowire mat. The side-view image clearly shows the preferential alignment of the nanowires along several crystallographic directions. In our experiments, the nanowires were pressed against a glass coverslip. This procedure increased the homogeneity of the layer and reduced the alignment (see Supplementary Section S1). Such compressed semiconductor nanowire mats were shown to be one of the most strongly scattering materials in the visible wavelength range $^{18}$.

\section{Characterization of diffuse transport}

Multiple scattering by the collective of nanowires - as compared to transport through individual nanowire waveguides - is characterized by a number of distinct features, such as diffuse broadening of the spatial intensity profile, coherent backscattering ${ }^{18}$, and spectral and spatial correlations. Several of these characteristics are shown in Fig. 1 and in the Supplementary Section S2. This includes the "memory effect", a shift of the intensity speckle pattern when displacing the sample over a distances less than the correlation length ${ }^{2}$. The total transmission $T$, shown in Fig. 1(b), indicates a strongly reduced transmission through nanowire mats of increasing thickness $L$, proportional to Ohms law $T \sim \ell / L$. Below $600 \mathrm{~nm}$ wavelength, the transmission is reduced by a tail of increasing absorption toward the band gap of $\mathrm{GaP}$ of $548 \mathrm{~nm}$. Above $600 \mathrm{~nm}$, the response is flat and indicative of low absorption losses in the nanowires. We have characterized the static and dynamic diffuse transport parameters of nanowire mats using a range of broadband methods ${ }^{18,19}$. Figure 1(c) shows the characteristic material parameters, mean free path $\ell$, diffusion constant $D$, and the transport velocity $v_{E}$ of light in the mat. The variation in $\ell$ and $D$ with wavelength indicates a transition from the weak scattering regime at long wavelengths to a resonant scattering regime at shorter wavelengths, characterized by a distribution of angle and wavelength dependent Leaky Mode Resonances, corresponding to the radial scattering modes of the cylindrical nanowires ${ }^{16,20}$. Evidence of anisotropic diffusion is observed as an ellipticity of the diffuse transmission (see Supplementary Section S2). Such anisotropy may help to lower the localization threshold ${ }^{21}$. The three-dimensional nature of the random medium can be inferred from the Thouless time of 1 ps obtained from the spectral correlation width shown further below, consistent with the diffuse transport time $L^{2} / D$ with $D$ the orientation- 
averaged diffusion constant. Quasi-ballistic transport in the vertical direction would give a transport time of around 20-40 fs for a $6 \mu \mathrm{m}$ thick slab. This result shows that the nanowire mat is an anisotropic three-dimensional random medium, in contrast to anisotropic media with one or more dimensions of decoupled quasi-ballistic transport ${ }^{22,23}$. The theoretical treatment of mesoscopic effects presented below disregards anisotropy or inhomogeneity along the thickness of the mat. While the general effect can be understood using available isotropic theory, in future work a fuller understanding might be obtained from models which take into account the sample geometry.

\section{Spatial intensity fluctuations}

To characterize mesoscopic light transport through nanowire mats we use the method of intensity fluctuations, which has been shown to be robust against absorption ${ }^{24,25}$. Enhanced intensity fluctuations are a result of the correlations induced by path crossings of the light inside the medium ${ }^{2}$. Theory for three dimensional media predicts increased fluctuations for reduced beam size, due to an increase of the crossing probability for two multiple scattering light paths ${ }^{12,26}$. Therefore, light was focused tightly onto the sample as shown in Fig. 2(a). The spatial intensity distribution at the surface of the nanowire mat was measured for many thousands of microscopic scattering configurations in order to obtain a statistically reliable data set (see Methods). The incident spot size was changed by displacing the illuminating microscope objective over a controlled distance, keeping the numerical aperture of illumination fixed.

Figure 2(b) and (c) show images of the normalized collected light $s_{a b}=t_{a b} /\left\langle t_{a b}\right\rangle$ for the 6 $\mu \mathrm{m}$ thick nanowire mat for illumination conditions "in focus" (IF) and "out of focus" (OF), corresponding to illumination spots of $1 \mu \mathrm{m}$ and $15 \mu \mathrm{m}$ width respectively (the same sample position was used for both measurements). While the out of focus images show a relatively homogeneous distribution of intensity fluctuations, many of the in focus images are governed by only one or a few bright transmission spots. Histograms of the normalized intensity $s_{a b}$ are shown in Fig. 2(d). The out of focus data is reasonably well described by a Rayleigh (negative exponential) distribution, representing the uncorrelated speckle fields ${ }^{2}$. A small deviation from the ideal exponent of -1 is observed due to limited experimental contrast (which also causes the small drop of the lowest intensities). However, even for out of focus illumination, 
an increased probability is found for high intensities $s_{a b}$. A fit using a mesoscopic transport model $^{27}$ gives good agreement for an out of focus conductance of $g^{\mathrm{OF}}=34 \pm 1$ (dashed red line). The histogram for in focus illumination shows a much larger deviation from the Rayleigh distribution, for which the mesoscopic model fit yields $g^{\mathrm{IF}}=3.6 \pm 0.1$ (black line). Figure 2(e) shows that the fits describe particularly well the lower moments of the distribution, which are generally used to quantify the mesoscopic contribution ${ }^{24,25}$. The low value of $g$ in focus indicates that on average only three independent open modes contribute to the intensity transmitted through the nanowire mat. This value is at least two orders smaller than values reported for other three-dimensional photonic materials ${ }^{12,13}$.

The dependence of the dimensionless conductance parameter $g$ on the incident beam width is shown in Fig. 2(e). The beam width as a function of focus displacement was independently calibrated using a GaP substrate without nanowires and modeled using Gaussian beam optics. For comparison, we also plot the focus dependence of $1 / \mathrm{g}$ for a nanowire mat of $1.6 \mu \mathrm{m}$ thickness. The fluctuations for the thinner sample are lower, indicating a lower contribution of mesoscopic interference corrections. An intuitive model for the dependence of $g$ on slab thickness and incident spot size is the "expanding waveguide" 12 . In this description a quasi-one dimensional expression for $g$ is integrated over short waveguide sections of increasing width at different depths inside the slab to describe the effect of diffuse spreading of the intensity (see Methods). The expanding waveguide model agrees well with diagrammatic theory for the intensity fluctuations and correlations ${ }^{13,26}$. As the probability of light paths crossing is inversely proportional to the waveguide width, mesoscopic interference effects occur predominantly in the first few mean free paths. It was found that the focus dependence of $g$ follows the expanding waveguide model well (lines in Fig. 2(f)), when taking into account a minimum width of the waveguide given by diffuse broadening of the illumination intensity over several mean free paths ${ }^{12}$. This broadening defines the smallest $g$ that can be measured. We note that our measured $g$ is much lower than the number of independent incident channels in the expanding waveguide which is of order $k_{\mathrm{GaP}}^{2} w^{2} \sim 10^{3}$, with $k_{\mathrm{GaP}} \simeq 33 \mu \mathrm{m}^{-1}$ the wavevector in the GaP substrate and $w$ the width of the diffuse input intensity.

The Supplementary Section S3 shows experimental results for light impinging from the top of the mat, obtained by reversing the direction of the optical beam in the setup. Qualitatively similar results are found as for illumination from the bottom. A deviation in value of 
$g^{\mathrm{IF}}$ might be caused by asymmetry of the nanowire mat, which tends to have a different morphology over its cross section (Fig. 1a), or by differences in the interface conditions (coverslip versus substrate).

\section{Total transmission fluctuations}

In addition to the spatial intensity statistics, we analyzed the fluctuations in the total transmission of light transmitted through the nanowire mat. Figure 3(a) shows an example of the transmission fluctuations as a function of sample position (see Methods), while the distribution $P\left(s_{a}\right)$ is shown in Figure 3(b). The out of focus histogram shows a narrow distribution which can be well described by a Gaussian (red curve). In comparison, the in focus histogram shows a pronounced broadening and an asymmetry with respect to a Gaussian (grey curve). These two characteristics indicate a deviation from the weak scattering regime. Random matrix theory has shown that the tail of the distribution can be described by an exponential $\sim \exp \left(-g s_{a}\right)^{27}$. Indeed a good fit is obtained for an exponential with a slope of $g=10.2 \pm 0.4$ (dashed line in Fig. 3(b)).

Figure 3(c) shows the experimental dependence of the total transmission fluctuations on beam width. Fully three-dimensional theory in the diffusion approximation ${ }^{26}$ (line) predicts a similar dependence of $\operatorname{var}\left(s_{a}\right)$ as our experimental data, however the variance lies systematically a factor 5.5 below the value expected from the intensity statistics according to $\operatorname{var}\left(s_{a b}\right)=1+2 \operatorname{var}\left(s_{a}\right)^{24}$. The measured variance is still considerably higher than the theoretical estimate for the fluctuation of the optical conductance $s=\sum_{a} s_{a}$ (red line $)^{28}$. At this point, no conclusive explanation can be given for this discrepancy between measured and theoretical $\operatorname{var}\left(s_{a}\right)$. An experimental limitation forms the selection of exactly one (spatial or angular) input channel, equivalent to a diffraction-limited focus. Typically, optical aberrations in the substrate and a limited numerical aperture are causes for deviations from perfect single-channel illumination. For illumination from the top (see Supplementary Section S3), we find a slightly better agreement, which supports the hypothesis of an experimental limitation.

In addition to experimental factors, the reduced variance may indicate limitations in the theoretical models linking $P\left(s_{a}\right)$ and $P\left(s_{a b}\right)$. We note in particular the extension of quasi one-dimensional models to three dimensions ${ }^{22}$, which may alter the relationship between 
the variances and the conductance parameter $g$, and an as yet unknown role of anisotropy. In addition, self-consistent localization theory has predicted significant deviations from the hallmark lognormal distribution in three dimensions, including a reduction of the variance and increased skewness ${ }^{29}$. Thus, the relationship between transmission fluctuations and $g$ is not absolutely established for such strongly scattering three-dimensional samples. Our results of Fig. 3 are the first experimental demonstration of a large deviation from the Gaussian distribution for total transmission in a three dimensional random medium. So far these fluctuations have been observed in the microwave regime ${ }^{24,25}$ or in a quasi-one dimensional geometry $^{22}$. While a deeper understanding requires a full theory including anisotropy, our results show that the regime of mesoscopic transport near the critical localization transition is experimentally accessible in three-dimensional nanowire mats.

Compared to the variance, the skewness (third cumulant) of the distribution, $\left(\left\langle s_{a}^{3}\right\rangle-\right.$ 1 ), is expected to be more robust against averaging over input channels, as it is entirely determined by contributions which include two path intersections ${ }^{13}$. We find a value for the skewness in focus of 0.08 for the distribution of Fig. 3(b), which is $10^{5}$ times larger than previous measurements of this quantity reported for slabs of $\mathrm{TiO}_{2}$ paint ${ }^{13}$. Using the relation $\left(\left\langle s^{3}\right\rangle-1\right)=16 / 45 g^{2}$ for three dimensional media in the diffusion approximation ${ }^{27}$ we find a value of $g^{\mathrm{IF}}=2.1$.

\section{Spatial and spectral correlations}

To complement our investigation into intensity fluctuations, we also examined the spatial and spectral correlations of the transmitted light induced by multiple scattering in nanowire mats (see Methods). These correlations form an important additional proof demonstrating that the observed fluctuations result from mesoscopic interference and not from sample inhomogeneity. The spatial $C(\Delta r)$ and spectral $C(\Delta \nu)$ correlations of the transmitted intensity are shown in Fig. 4(a-c). Both the out of focus spatial and spectral correlations could be fitted using only a short-range $\left(C^{(1)}\right)$ contribution (red lines), where the spectral correlation returned a value for the diffusion constant $D$ of $14 \pm 1 \mathrm{~m}^{2} \mathrm{~s}^{-1}$. The in focus spatial and spectral correlations show a marked increase in both amplitude and width because of the presence of additional long-range correlations ${ }^{2}$. For the spatial correlation, the long-range component was obtained by subtracting the two correlations in and out of focus, yielding 
a typical $1 / \Delta r$ decay that could be fitted using $g=3.0 \pm 0.1$. The spectral correlation in Fig. 4(b) also exhibits long range behavior, which is characterized by a decay proportional to $\Delta \nu^{-0.52}$. In addition, a long-range spectral correlation was found in focus for the total transmission (see Supplementary Section S5). These correlations confirm the general picture of the statistical analysis, namely, that transport of light through nanowire mats is highly correlated and characterized by only a small number of open transmission channels.

We emphasize that one cannot directly compare the low number of open modes to the Thouless criterium for wave localization ${ }^{25}, g=1$. The effective number of open modes $g$ in the expanding waveguide can be lower than 1; however, these are only the modes selected by the illumination spot. Other modes are present outside this region which may still spectrally overlap in the three-dimensional medium, even if they are not excited in our experiment. Still, the results for focused illumination are significant as the enhanced mesoscopic interference enables new quantifications of the proximity of the localization threshold. For example, in the Supplementary Section S4 we have presented distributions for the same nanowire mat after infiltration with a transparent epoxy. Immersion with a high refractive index epoxy results in a lower scattering strength of the nanowire mat, leading to a reduction of the crossing probability and a suppression of fluctuations to a value of $g^{\mathrm{IF}}=15$ and $g^{\mathrm{OF}} \simeq 950$.

\section{Conclusions}

The high-density semiconductor nanowire mats in this study exhibit very strong interference contributions caused by mesoscopic transport. Statistical analysis of intensity fluctuations shows that transport for focused illumination is governed by a minimum of around 3 open transmission modes, which is a record low value for light in a three-dimensional medium. These effects are so strong that we observe a change in the distribution from Gaussian towards a skewed distribution that is predicted in the theory but never previously seen in a three-dimensional optical medium. These observations are backed up by the spectral and spatial correlations of the transmitted light, which exhibit much stronger long-range correlation than found in other three-dimensional photonic media. The presence of strongly correlated transport shows that traditional light diffusion models are no longer valid when describing photon transport and emission in strongly scattering nanowire mats, which is of importance for a wide range of applications. Future studies are needed to investigate how 
the collective scattering affects, for example, the emission of light in nanowire mats for applications in LEDs, or the absorption in light harvesting devices. Such studies may explore how mesoscopic transport and long-range correlation can lead to new regimes of light trapping ${ }^{30}$. Ultimately, the transition to Anderson localization could lead to the optical equivalent of the metal to insulator transition in electronics. While significant further optimizations of the semiconductor nanowire mats are required, we believe that this regime appears to be within reach of future experiments.

\section{METHODS}

Fabrication of nanowire mats. Mats of gallium phosphide (GaP) nanowires were synthesized using metal-organic vapour-phase epitaxy employing a multi-step approach developed in Ref. 18. In this method, vapor-liquid-solid growth is altered with radial growth in order to grow high-density arrays of nanowires of 100-150 nm diameter with volume fraction around $50 \%$. By repeating this procedure several times, thick wire mats could be reproducibly achieved over large areas with a high uniformity. The nanowires were pressed against a glass coverslip of $170 \mu \mathrm{m}$ thickness, where a colour change was observed indicating good mechanical contact of the wires and the glass.

Optical experiments. For the characterization of transmission modes we use the statistical approach developed by Genack and co-workers ${ }^{24}$. Light from a HeNe laser $(632.8 \mathrm{~nm}$ wavelength) was focused onto the nanowire slab through the GaP substrate by using a 0.9 N.A. microscope objective with a working distance of $1.0 \mathrm{~mm}$ (Nikon CFI60 LU Plan BD). The transmitted intensity at the exit plane was collected by a 1.3 N.A. oil-immersion objective with cover-slip correction (Nikon CFI60 Plan Fluor) and imaged onto a 16-bit monochrome CMOS camera (Allied Vision Technology). For each statistical average, between 2,000 and 20,000 different images were obtained by translating the sample in the focal plane using a motorized stage. As the amount of coherence areas scales with the spot size, more images were required for the in focus than for the out of focus statistics.

An important issue for real samples is to discriminate statistical fluctuations originating from mesoscopic effects from those caused by local sample inhomogeneity. It was found that sample variations had a much longer length scale (tens of $\mu \mathrm{m}$ ) than the typical sub- $\mu \mathrm{m}$ range of mesoscopic effects, and so these variations could be effectively divided out by a moving 
average of the total transmission. Fluctuations in the laser intensity amounted to no more than $\pm 2 \%$ and of long enough range to be removed by the moving average.

Each image was normalized to the ensemble averaged image in order to obtain the normalized transmission coefficient $s_{a b}=t_{a b} /\left\langle t_{a b}\right\rangle$. The brackets denote the ensemble average. Only the center area of the spot corresponding to the half-maximum of the average spot were included in the analysis of $s_{a b}$ to minimize the effect of the background. An area out to $13.5 \%$ of the average was analyzed for the total transmission, corresponding to a $1 / e^{2}$ cut-off. Experimental contrast was included as a prefactor that affected the average image. Spectral correlations were obtained using a external cavity stabilized tunable diode laser (Thorlabs) with a center wavelength of $770 \mathrm{~nm}$, linewidth not exceeding $130 \mathrm{KHz}$ and a tuning range of approximately $20 \mathrm{~nm}$. The contrast was reduced to 0.8 , mainly because of the increased width of the point spread function at longer wavelength (diffraction limit), while the spatial correlation width remained approximately constant.

Analysis. Statistical distributions were fitted to a mesoscopic model of light transport by Nieuwenhuizen and Van Rossum ${ }^{27}$. The limited experimental contrast is included as as a pre factor to the average intensity. The tail of the in focus distribution follows well the exact limiting behavior, given by the stretched exponential $P\left(s_{a b}\right) \propto \exp \left(-2 \sqrt{g s_{a b}}\right)$. For the expanding waveguide model, we followed the method by Scheffold et al. ${ }^{12}$ to define an effective conductance parameter $g$ using an integral over infinitesimally short waveguide sections of linearly expanding width in the vertical $z$ direction, resulting in the final expression

$$
1 / g=\int_{0}^{L} d z 1 / g(z)=\frac{3 L}{k^{2} w^{2} \ell} F\left(\frac{w}{L}\right)
$$

with

$$
F\left(\frac{w}{L}\right)=\int_{0}^{\infty} d x\left(\frac{w}{L}\right)^{2} \exp \left[-\left(\frac{w}{L}\right)^{2} \frac{x^{2}}{32}\right] \frac{x(\sinh (x) / x-1)}{8(\cosh (x)-1)},
$$

which corresponds to Eq. (32) of Pnini and Shapiro for a Gaussian illumination profile ${ }^{26}$. The function $F(x)$ has limiting behavior $2 x / 3$ for $x<<1$ and $2 / 3$ for $x>>1$. Here $w$ corresponds to the initial width of the waveguide $w \simeq w_{0}+2.4 \ell$, where $w_{0}$ is the size of the incident light and $2.4 \ell$ is the approximate diffuse broadening of the intensity. For the theoretical estimate of $\operatorname{var}\left(s_{a}\right)$ we made use of the expression $\operatorname{var}\left(s_{a}\right)=2 / 3 g$ using the $g$ obtained from the fits of $P\left(s_{a b}\right)$. The optical conductance fluctuations were obtained for the same value of $g$ using the result by Van Rossum et al. for a three dimensional medium ${ }^{28}$

$$
\operatorname{var}(s)=\frac{9}{2 \pi k^{4} w^{2} \ell^{2}}
$$


The spatial and spectral intensity-intensity correlation functions were fitted using wellknown expressions in the diffusion approximation, see e.g. ${ }^{2}$. Traditionally, one distinguishes in the correlation function different components $C=C^{(1)}+(1 / g) C^{(2)}+\left(1 / g^{2}\right) C^{(3)}$. $C^{(1)}$ defines the width of the speckle defined by interference outside the medium, while $C^{(2)}$ and $C^{(3)}$ are corrections due to one and two crossings of light paths, respectively, inside the medium $^{2}$. Intuitively, one can understand these correlations from the fact that at a crossing, information about the origin of the light paths is lost and waves appearing from different directions propagate together without losing phase coherence. Dephasing only takes place in the segments before the crossing and correlations become long-range ${ }^{2}$. For the long-range spatial correlation we use form of the $C^{(2)}(\Delta r) \propto L / \Delta r$ for $\Delta r>\ell$, interpolated to the value of $4 / 3 g$ for $\Delta r=0$.

1 Beenakker, C.W.J. Random-matrix theory of quantum transport, Rev. Mod. Phys. 69, 731-808 (1997).

2 Akkermans E. \& Montambaux G., Mesoscopic Physics of Electrons and Photons, Cambridge University Press, 427-464 (2007).

3 Popoff, S.M., Lerosey, G., Carminati, R., Fink, M., Boccara, A.C. \& Gigan, S. Measuring the Transmission Matrix in Optics: An Approach to the Study and Control of Light Propagation in Disordered Media, Phys. Rev. Lett. 104, 100601 (2010).

4 Mosk, A.P., Lagendijk, A., Lerosey, G. \& Fink, M., Controlling waves in space and time for imaging and focusing in complex media Nat. Photon. 6, 283-292 (2012)

${ }^{5}$ Kim, M., Choi, Y., Yoon, C., Choi, W., Kim, J., Park Q.-H. \& Choi, W. Maximal energy transport through disordered media with the implementation of transmission eigenchannels, Nat. Photon. 6, 581-585 (2012).

${ }^{6}$ Lagendijk, A., Van Tiggelen, B.A. \& Wiersma, D.S. Fifty-years of Anderson localization, Phys. Today 62, 24-29 (2009).

7 Strybulevych, A., Hu, H., Page, J.H., Skipetrov, S.E. \& van Tiggelen, B.A. Localization of ultrasound in a three-dimensional elastic network, Nat. Phys. 4, 945-948 (2008).

8 Jendrzejewski, F., Bernard, A., Müller, K., Cheinet, P., Josse, V., Piraud, M., Pezz, L., SanchezPalencia, L., Aspect A. \& Bouyer, P. Three-dimensional localization of ultracold atoms in an 
optical disordered potential, Nat. Phys. 8, 398-403 (2012).

9 Wiersma, D.S., Bartolini, P., Lagendijk, A. \& Righini. R. Localization of light in a disordered medium, Nature 390,671-673 (1997).

10 Störzer, M., Gross, P., Aegerter, C.M. \& Maret, G. Observation of the Critical Regime Near Anderson Localization of Light, Phys. Rev. Lett. 96, 063904 (2006).

11 Van der Beek, T., Barthelemy, P., Johnson, P.M., Wiersma D.S. \& Lagendijk A. Light transport through disordered layers of dense gallium arsenide submicron particles, Phys. Rev. B 85, 115401 (2012)

12 Scheffold F., Härtl, W., Maret, G. \& Matjevic, E. Observation of long-range correlations in temporal intensity fluctuations of light, Phys. Rev. B56,10942-10952 (1997).

13 De Boer, J.F., Van Rossum, M.C.W., Van Albada, M.P., Nieuwenhuizen T.M. \& Lagendijk, A., Probability distribution of multiple scattered light measured in total transmission, Phys. Rev. Lett. 73(19), 2567-2570 (1994).

14 Yan, R., Gargas, D. \& Yang, P. Nanowire photonics, Nat. Photon. 3, 569 - 576 (2009).

15 Garnett, E. \& Yang, P. Light Trapping in Silicon Nanowire Solar Cells, Nano Lett. 10(3), 1082-1087.

16 Cao, L., White, J.S., Park J.-S., Schuller, J.A., Clemens B.M. \& Brongersma, M.L. Engineering light absorption in semiconductor nanowire devices, Nat. Mater. 8, 643-647 (2009).

17 Wallentin J., Anttu N., Asoli D., Huffman M., Aberg I., Magnusson M.H., Siefer G., FussKailuweit P., Dimroth F., Witzigmann B., Xu H. Q., Samuelson L., Deppert K., \& Borgström M. T. InP Nanowire Array Solar Cells Achieving 13.8\% Efficiency by Exceeding the Ray Optics Limit, Science DOI: 10.1126/science.1230969 (2013)

18 Muskens, O.L., Diedenhofen, S.L., Kaas, B.C., Algra, R.E., Bakkers, E.P.A.M., Rivas, J.G. \& Lagendijk, A. Large Photonic Strength of Highly Tunable Resonant Nanowire Materials, Nano Lett. 9(3),930-934 (2009)

19 Muskens, O.L. \& Lagendijk A., Method for broadband spectroscopy of light transport through opaque scattering media, Opt. Lett. 34, 395-397 (2009).

20 Brönstrup, G., Jahr, N., Leiterer, C., Csaki, A., Fritzsche, W. \& Christiansen, S. Optical Properties of Individual Silicon Nanowires for Photonic Devices, ACS Nano 4(12), 7113-7122 (2010).

21 Kaas, B., van Tiggelen, B., \& Lagendijk, A., Anisotropy and interference in wave transport: 
An analytic theory, Phys. Rev. Lett. 100, 243901 (2008).

22 Park J., Zhang S., Genack A.Z., Intensity statistics and photon localization beyond one dimension, Phys. Rev. E 82 045101(R) (2010)

23 Levi, L., Krivolapov, Y., Fishman, S. \& Segev, M. Hyper-transport of light and stochastic acceleration by evolving disorder, Nature Physics 8, 912 (2012).

24 Chabanov, A.A., Stoytchev M. \& Genack A.Z. Statistical signatures of photon localization Nature 404, 850-853 (2000).

25 Genack A.Z. \& Chabanov A.A. Signatures of photon localization, J. Phys. A: Math. Gen. 38, $10465-10488$ (2005).

26 Pnini R. \& Shapiro, B. Fluctuations in transmission of waves through disordered slabs, Phys. Rev. B 39(10), 6986-6994 (1989).

27 Nieuwenhuizen, T.M. \& van Rossum, M.C.W., Intensity distributions of waves transmitted through a multiple scattering medium, Phys. Rev. Lett. 74, 2674-2677 (1995).

28 van Rossum M.C.W., Nieuwenhuizen T.M., \& Vlaming R. Optical conductance fluctuations: Diagrammatic analysis in the Landauer approach and nonuniversal effects, Phys. Rev. E 51 6158-6176 (1995)

29 Muttalib K.A., Markoš P.,Wölfe P., Conductance distribution in strongly disordered mesoscopic systems in three dimensions, Phys. Rev. B 72, 12317 (2005)

30 Vynck, K., Burresi, M., Riboli. F. \& Wiersma, D.S. Photon management in two-dimensional disordered media, Nat. Mater. 11, 1017-1022 (2012).

\section{Acknowledgements}

The authors thank A. Mosk of the University of Twente and A. Lagendijk of the FOMInstitute AMOLF for valuable discussions. The research leading to these results has received funding from the European Union Seventh Framework Programme under grant agreement no. 265073. O.L.M. acknowledges financial support from EPSRC grant EP/J016918/1, from the Royal Society through an International Joint Project, and from the EU Network of Excellence "Nanophotonics for Energy Efficiency" (N4E). 
a

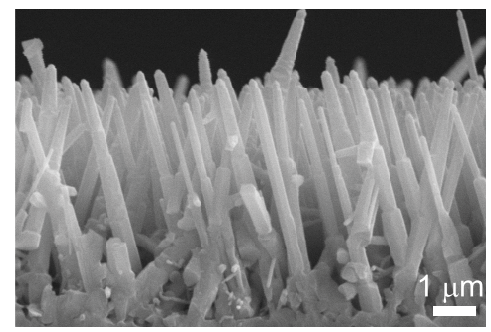

b

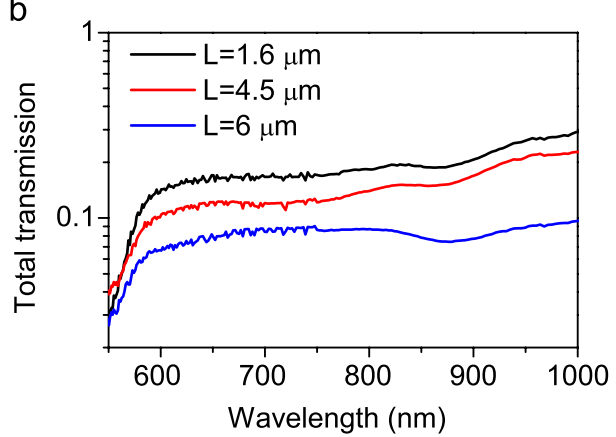

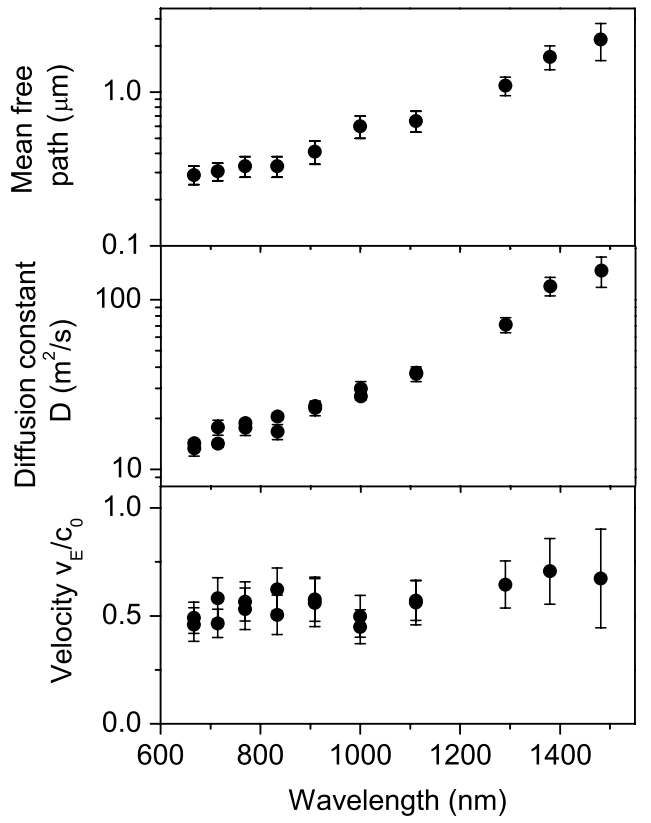

FIG. 1: Morphology and basic light transport parameters reveal strong multiple scattering in nanowire mats. (a) Side-view scanning electron microscopy image of a cleaved section of the $6 \mu \mathrm{m}$ thick nanowire mat, showing the orientation of the nanowires before compression. (b) Spectral dependence of total transmission of light through nanowire mats of three different thicknesses $L$, along with (c) transport mean free path $\ell$, diffusion constant $D$ and energy velocity $v_{E}$ for the nanowire mat obtained from broadband characterization methods ${ }^{19}$.

\section{Author contributions}

O.M. and T.S. conceived the idea and designed the experiments, C.B. and T.S. constructed the setup, performed the experiments and data analysis. T.Z. fabricated and characterized the nanowire mats. O.M. and E.B. coordinated and directed the study. All authors contributed to manuscript preparation, data interpretation, and discussed the results.

\section{Competing financial interests}

The authors declare no competing financial interests. 


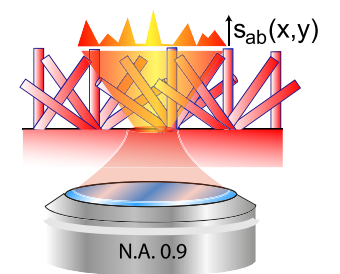

d

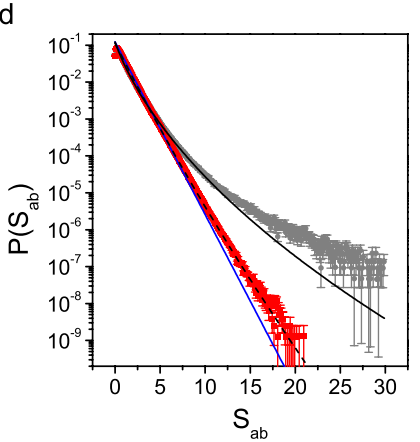

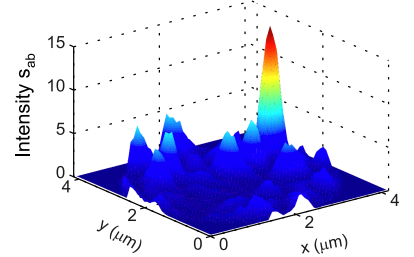

e

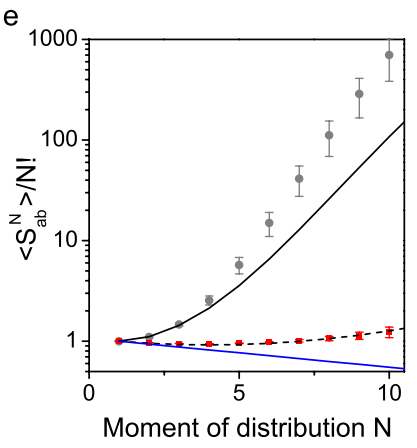

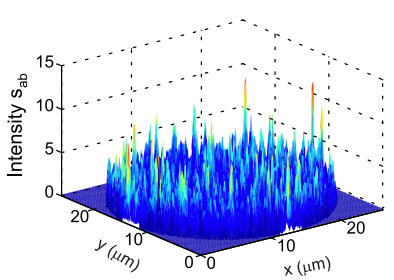

$f$

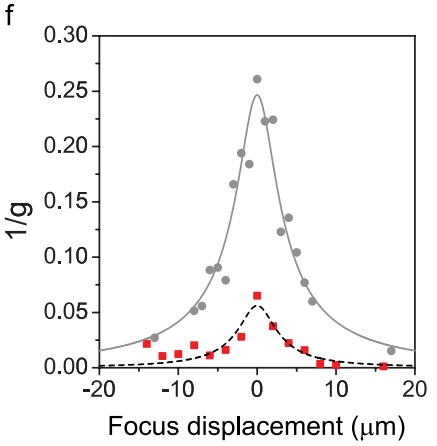

FIG. 2: Enhanced spatial intensity fluctuations in the optical transmission through nanowire mats are explained by a small number of open channels. (a) Schematic of light transport through a nanowire mat. (b,c) Normalized transmission at same point on sample in and out of focus respectively. (c,d) Histogram of intensity distribution $P\left(s_{a b}\right)$ for $6-\mu \mathrm{m}$ thick nanowire mat and corresponding moment distribution. Grey points are in focus (IF), red points out of focus (OF). Grey and red lines are fits using a mesoscopic model ${ }^{27}$, yielding $g^{\mathrm{IF}}=3.6 \pm 0.1$, and $g^{\mathrm{OF}}=34 \pm 1$ respectively. Blue line is a fit using negative exponential Rayleigh-statistics with a contrast of 0.96. Distributions are characterized by $\operatorname{var}\left(s_{a b}^{\mathrm{IF}}\right)=1.204, \operatorname{var}\left(s_{a b}^{\mathrm{OF}}\right)=0.937$, $\operatorname{var}\left(s_{a b}^{\text {Rayleigh }}\right)=0.921$.(e) Calculated moments of the experimental distributions and their fits. (f) Dependence of $1 / g$ on vertical translation from focus, obtained from fits for the 6 - $\mu \mathrm{m}$ thick sample (grey dots) and for a $1.6 \mu \mathrm{m}$ thick mat (red squares). Lines are fits using the expanding waveguide model $^{12}$. 

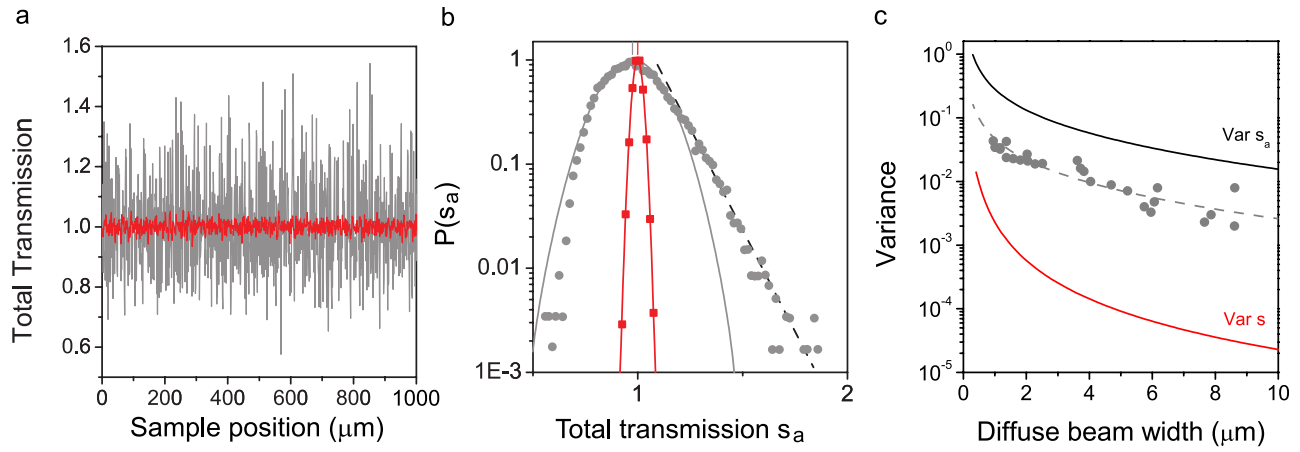

FIG. 3: Total transmission measurements show a significant deviation from Gaussian statistics. (a) Total transmission as a function of position for the $6-\mu \mathrm{m}$ sample, normalized to a 10- $\mu \mathrm{m}$ moving average. Grey lines are for in focus, red lines for out of focus illumination. (b) Histogram of the total transmission distribution $P\left(s_{a}\right)$. The distributions are characterized by variances $\operatorname{var}\left(s_{a}^{\mathrm{IF}}\right)=0.025$ (grey line) and $\operatorname{var}\left(s_{a}^{\mathrm{OF}}\right)=4.2 \times 10^{-4}$ (red line). Lines are Gaussian fits; the peak of each fit is marked by a line of corresponding color. Dashed line indicates exponential tail fit $\sim \exp \left(-g s_{a}\right)$ with $g=10.2 \pm 0.4$. (c) Variance of the $P\left(s_{a}\right)$ distribution versus beam width (points), with theoretical calculations for $\operatorname{var}\left(s_{a}\right)^{26}$ and $\operatorname{var}(s)^{28}$ in the diffusion approximation. Dashed line: $\left[\operatorname{var}\left(s_{a}\right)\right] / N$ with $N=5.5$ the experimental reduction of the variance.
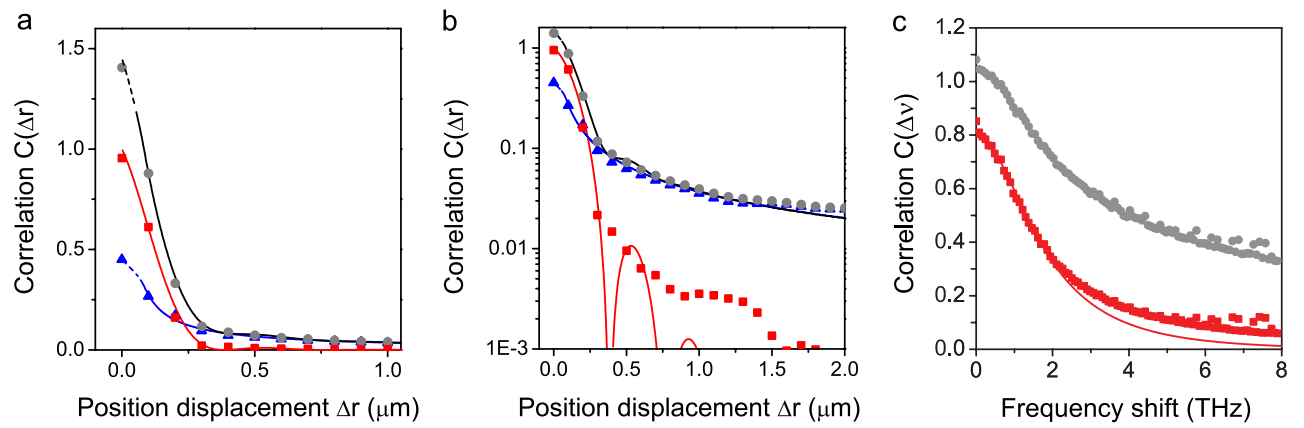

FIG. 4: The mesoscopic regime is confirmed by observation of long-range spatial and spectral correlations. Spatial correlation on a linear (a) and logarithmic (b) scale. Grey points are in focus, red points are out of focus. Blue triangles: long-range contribution obtained by subtracting in and out of focus data. Lines: fits using $C^{(1)}(\Delta r)$ and $C^{(2)}(\Delta r)^{2}$; dash indicates extrapolation from $1 / \Delta r$ for $\Delta r>\ell$ toward $4 / 3 g$ for $\Delta=0$. (c) Spectral correlation obtained using tunable diode laser around $770 \mathrm{~nm}$ wavelength, with (red line) fit using $C^{(1)}(\Delta \nu)$. 